\title{
Design and Application of Feed Water Control System for Super-critical Power Generation Unit
}

\author{
HAN Zhongxu \\ China Electric Power Research Institute \\ Beijing, China \\ zhongxuh@epri.sgcc.com.cn
}

\author{
TIAN Xinshou \\ China Electric Power Research Institute \& North China \\ Electric Power University \\ Beijing, China \\ tianxinshou@epri.sgcc.com.cn
}

\begin{abstract}
The characteristics of super-critical power generation unit coordinated and feed water control system are strong coupling of multiple inputs and multiple outputs, nonlinearity, time-varying parameters. Design object of the study work is that controlled systems are controllable and stable. Two type of the practical running power were used as research object, the control system was designed accordingly. At first, the preconditions and proofs of stability of coordinated control system (CCS) and feed water control system (FWCS) were given respectively, then stability analysis for CCS and FWCS were made by mathematical derivation according to the technical problems of FWCS. The application results of super critical power unit show effectiveness and practicability.
\end{abstract}

Keywords-supercritical pressure; once-through boiler; boilerturbine coordinated control system; boiler feed water control; non-linear; stabilization; state feedback control

\section{INTRODUCTION}

The improvement of the steam parameters for power generation unit makes the density difference between water and steam decrease, and the natural circulation of boiler is restricted. There is a pressure instability region, when the boiler operates at the vicinity of critical pressure[1], so the super-critical power generation unit is always composed with once-through boiler.

There is no fixed boundary in the heating surface among feed water heating, evaporation and overheating process of once-through boiler ${ }^{[1-2]}$. The evaporation is equal to the feed water, and the superheated steam temperature depends on the ratio of feed water and the heat provided by feed fuel in the stable conditions. The superheated steam temperature at boiler outlet is essentially unchanged, when superheated steam temperature changed at certain proportion. The main method that once-through boiler control superheated steam temperature ensures that the ratio of feed water and the heat provided by feed fuel keep within a certain range, but the desuperheating spray based on the method went to fine-tune steam temperature.

Because the caloric value of fuel flow is difficult to realtime measure online, the method that fuel-water ratio controls feed water flow is always be used, so the change of fuel characteristics or mixed between different fuel characteristics makes the accuracy and effectiveness of fuelwater ratio adjust affected in a certain extent. Besides, the response delay of outlet temperature is big when the change of fuel-water ratio, so the outlet temperature as feedback of fuel-water ratio adjust is impossible ${ }^{[3]}$.

The paper based on the research about mathematical model of controlled object ${ }^{[4-6]}$ study the interaction between feed water and coordinated control system of power generation unit according to the technical problems of feed water control system for supercritical power generation unit. Two type of the practical running power were used as research object, the control system was designed accordingly. Then the conditions of stability for CCS and FWCS were made by mathematical derivation, and the application results of super-critical power unit show effectiveness of the method.

\section{DESIGN OF COORDINATED AND FEED WATER CONTROL SYSTEM FOR SUPER-CRITICAL POWER GENERATION UNIT}

When the heat load is fixed, and feed water is decline, then the section of heating and evaporation become shorter, and the section of overheating becomes length, which makes the superheated steam temperature rising according to oncethrough boiler and the result is opposite if the feed water flow increasing. Therefore, in order to making the oncethrough boiler has a good static and dynamic regulation characteristics, an excellent feed water control system is applied.

The coordinated and feed water control system for supercritical power unit as follow figure1.

Because the super-critical power generation unit have not drum, the main different ${ }^{[8]}$ of coordinated control system between once-through boiler and drum-boiler ${ }^{[7]}$ as follows:

1) The caloric value corrected loop is designed in fuel control loop of boiler, thet BTU caloric value compensate[9]. So that the coordinated system between boiler-turbine coordination and feed water control system is designed through the demand of heat rather than the simple mixed of fuel-water ratio.

2) Considering the effect of BTU caloric value compensation in the designed loop of increment function observer (IFO-K $\Delta \mathrm{x}^{[10]}$ ).

3) The two levels accelerate loop are designed in the main control loop of boiler, and the second accelerate loop considers the impact of various operating conditions including pressure deviation. 


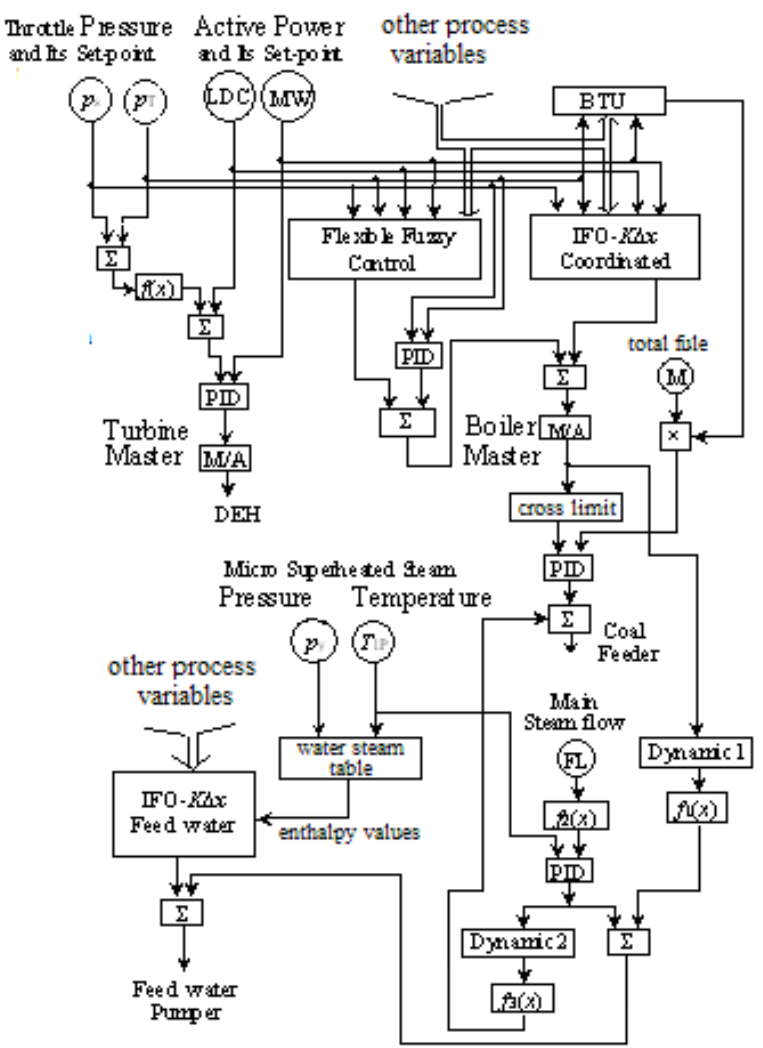

Figure 1. Schematic diagram of coordinated and feed water control system for super-critical power unit

The heat injected into the boiler is suitable at different operating conditions, because the coordinated control system considers BTU caloric value compensation.

The designed scheme of feed water control system as follows: The main controller of feed water control loop forms feed water flow command through receiving the feed water estimation. The deviation between feed water flow command and measured feed water is calculated by PID regulator laws to adjust the speed of the pump through controller output.

The characteristics of feed water flow loop as follows:

1) The mathematical expectation is obtained based on actual correspondence between fuel flow and feed water flow in the long operating using probability statistics method, then the functional relationship $\mathrm{f}_{1}(\mathrm{x})$ between fuel flow (BTU compensate) and feed water flow under different loads is adjustted.

2) Similarly, the mathematical expectation of separator outlet steam temperature under different loads, that the functional relationship $\mathrm{f}_{2}(\mathrm{x})$ between steam flow and separator outlet steam temperature is adjustted.

3) In order to predict the trends of feed water flow, and correct feed water flow command, the state feedback control based on IFO-K $\Delta \mathrm{x}$ is introduced.
4) The deviation between separator outlet steam temperature and estimation is regulated by PID to correct feed water flow estimation.

5) The dynamic process one is introduced to the coordinated loop between fuel flow and feed water flow to compensate dynamic deviation between fuel flow and feed water flow.

The function of dynamic process two and $f_{3}(x)$ is similar with reducer, the increasing (decreasing) of fuel flow is restricted, when the separator outlet steam temperature increase (decrease) fast.

If the separator outlet steam temperature is effectively controlled in the above designed scheme, the effect of superheated steam temperate control is smaller. The control scheme uses separator outlet steam temperature as the characterization of energy balance between input heat and absorbing heat. The driving variation is main control output of boiler, and the passive variation is feed water flow, and the output of separator outlet steam temperature controller as feed water corrected signal in the ratio control part.

\section{STABILITY ANALYSIS FOR CONTROL SYSTEM}

An observed system is n-dimensional, controllable, linear, slow time-variant and multi-output.

$$
\begin{cases}\dot{\boldsymbol{x}} & =\boldsymbol{A}(t) \boldsymbol{x}+\boldsymbol{B}(t) \boldsymbol{u}, \boldsymbol{x}\left(t_{0}\right)=\boldsymbol{x}_{\boldsymbol{0}} \\ \boldsymbol{y} & =\boldsymbol{C}(t) \boldsymbol{x}\end{cases}
$$

The time-variant characteristics Hammerstein model ${ }^{[12]}$ can be expressed by it and a group high order polynomial which represents nonlinear gain simultaneously.

$$
\left\{\begin{array}{l}
u=f_{v_{1}}\left(v_{1}\right)=f_{v}(v) \\
v_{1}=k_{\mathrm{BTU}} \cdot v
\end{array}\right.
$$

$\boldsymbol{x} \in \boldsymbol{R}^{n} ; \boldsymbol{A}(t) 、 \boldsymbol{B}(t) 、 \boldsymbol{C}(t)$ in formula are proper order time-variant real matrix; $\boldsymbol{k}_{\mathrm{BTU}}$ is a proper order diagonal matrix, which express some uncertain factor(for example caloric value of fuel), and the state equation as follows:

$$
\left\{\begin{aligned}
\dot{\boldsymbol{z}} & =\boldsymbol{F} \boldsymbol{z}+\boldsymbol{G} \Delta \hat{\boldsymbol{y}}+\boldsymbol{H} \Delta \hat{\boldsymbol{u}}, \quad \boldsymbol{z}\left(t_{\boldsymbol{o}}\right)=\boldsymbol{z}_{0} \\
\boldsymbol{w} & =\boldsymbol{M} \boldsymbol{z}+\boldsymbol{N} \Delta \hat{\boldsymbol{y}} \\
\Delta \hat{\boldsymbol{u}} & =\boldsymbol{v}-\boldsymbol{f}_{\boldsymbol{v}}\left(\boldsymbol{R}_{D M D}\right) \\
& =\tilde{\boldsymbol{k}}_{\mathrm{BTU}} \cdot \boldsymbol{v}-\boldsymbol{f}_{\boldsymbol{v}_{1}}\left(\boldsymbol{R}_{D M D}\right) \\
\Delta \hat{\boldsymbol{y}} & =\boldsymbol{y}-\boldsymbol{f}_{\boldsymbol{y}}\left(\boldsymbol{R}_{D M D}\right)
\end{aligned}\right.
$$

That can express a simultaneous system IFO-K $\Delta \mathrm{x}^{[8,10]}$.

There is a following theorem[8], if the effect of feed water control system to boiler-turbine coordinated control system do not be considered.

Lemma1: the synthetic coordinated control system based on state back control IFO-K $\Delta \mathrm{x}$ of formula(3) is composed with controlled object of observability simultaneous system(1)and(2) and simultaneous system(4)-(8), and the close loop-control is asymptotical stabilization, if it meets the following sufficient condition.

(1) $\operatorname{Re} \lambda(\boldsymbol{F})<0$ 
(2)

(2) $\left\{\begin{array}{l}\lim _{t \rightarrow \infty} \Delta \hat{\boldsymbol{u}}=\lim _{t \rightarrow \infty}\left[\tilde{\boldsymbol{k}}_{B T U} \cdot \boldsymbol{v}-\boldsymbol{f}_{\boldsymbol{v}_{1}}\left(\boldsymbol{R}_{D M D}\right)\right]=\xi_{1},\left\|\xi_{1}\right\|_{\infty} \leq \omega_{1} \\ \lim _{t \rightarrow \infty} \boldsymbol{v}=\gamma\end{array}\right.$

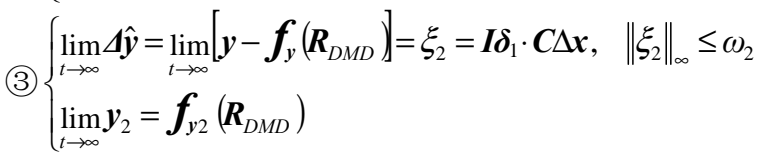

(4) $\alpha[\boldsymbol{A}(t)]=0$ and $\alpha(\boldsymbol{F})$ are non-singular(there is no public eigenvalue between matrix $\boldsymbol{A}(t)$ and $\boldsymbol{F}$ at any moment)

(5) $\{\boldsymbol{A}(t), \boldsymbol{C}(t)\}$ is complete observability and $\{\boldsymbol{F}, \boldsymbol{G}\}$ is complete controllability at any moment

(6) And the proper order time-variant matrix $\boldsymbol{T}(t)$ which meets the following formula is existence.

$$
\boldsymbol{M T}(t)+N C(t)=\boldsymbol{K}(t)
$$

(7) $\lim _{t \rightarrow \infty} \boldsymbol{f}_{w 1}\left(\hat{\boldsymbol{y}}, \boldsymbol{R}_{D M D}\right)=\boldsymbol{f}_{v_{1}}\left(\boldsymbol{R}_{D M D}\right)+\lim _{t \rightarrow \infty} \Delta \hat{\boldsymbol{u}}$

(8) $\lim _{t \rightarrow \infty}\left(\boldsymbol{k}_{\mathrm{BTU}}-\tilde{\boldsymbol{k}}_{\mathrm{BTU}}\right)=\boldsymbol{\xi}_{3}, \quad\left\|\boldsymbol{\xi}_{3}\right\|_{*} \leq \omega_{3}$

(9) $\lim _{t \rightarrow \infty} \Delta \hat{\boldsymbol{y}}_{2 P I D}=-\boldsymbol{M}\left(\boldsymbol{F}^{-1} \boldsymbol{H} \boldsymbol{I} \xi_{1}+\boldsymbol{F}^{-1} \boldsymbol{G} \boldsymbol{I} \boldsymbol{\xi}_{2}\right)+\boldsymbol{N} \Delta \hat{\boldsymbol{y}}+\boldsymbol{\xi}_{3} \cdot \boldsymbol{v}$

$\xi_{1}, \gamma, \xi_{2}, \boldsymbol{\delta}_{1}$ are proper dimensional real vector, $\boldsymbol{\xi}_{3}$ is proper dimensional real matrix, $\omega_{1}, \omega_{2}, \omega_{3}$ are proper small real numbers, $\left\|\boldsymbol{\xi}_{1}\right\|_{\infty},\left\|\boldsymbol{\xi}_{2}\right\|_{\infty}$ express maximal norms ${ }^{[13]}$ of vector $\boldsymbol{\xi}_{1}$ and $\boldsymbol{\xi}_{2}$ respectively. ${ }^{*}=1,2$ or $\infty$ in $\left\|\boldsymbol{\xi}_{3}\right\|_{*}$, which express the row norm, spectral norm, as well as columns norm ${ }^{[13,14]}$ of matrix $\boldsymbol{\xi}_{3}, \widetilde{\boldsymbol{k}}_{\text {BTU }}$ is estimation ${ }^{[9]}$ of caloric value observer. The other matrix and variable descript state space of controlled object and observer ${ }^{[14]}$.

The associate regulator of serial regulation system as follows:

$$
\left\{\begin{array}{l}
-\Delta \hat{\boldsymbol{y}}_{1 P I D}=\boldsymbol{I} \boldsymbol{\beta}_{1} \cdot \Delta \hat{\boldsymbol{y}}_{1}+\boldsymbol{I} \boldsymbol{\beta}_{2} \cdot \boldsymbol{z}_{1}+\boldsymbol{I} \boldsymbol{\beta}_{3} \cdot \Delta \dot{\hat{\boldsymbol{y}}_{1}} \\
\dot{\boldsymbol{z}}_{1}=(k+1) \cdot \Delta \hat{\mathbf{y}}_{1}, \quad \boldsymbol{z}_{1}\left(t_{\boldsymbol{0}}\right)=\boldsymbol{z}_{10}
\end{array}\right.
$$

The main regulator of controlled object in boiler-turbine coordinated control system as follows:

$$
\left\{\begin{array}{l}
-\Delta \hat{\boldsymbol{y}}_{2 P I D}=\boldsymbol{I} \beta_{4} \cdot \boldsymbol{\Delta} \hat{\boldsymbol{y}}_{2}+\boldsymbol{I} \boldsymbol{\beta}_{5} \cdot \boldsymbol{z}_{2}+\boldsymbol{I} \boldsymbol{\beta}_{6} \cdot \Delta \dot{\boldsymbol{y}} \dot{\hat{y}}_{2} \\
\dot{\boldsymbol{z}}_{2}=(k+1) \cdot \boldsymbol{\Delta} \hat{\boldsymbol{y}}_{2}, \quad \boldsymbol{z}_{2}\left(t_{\boldsymbol{o}}\right)=\boldsymbol{z}_{20}
\end{array}\right.
$$

The different algorithms which can improve the speed of control system are represented by the following formula, for example the control of feed-forward, prediction and analog flexible $^{[15]}$.

$$
\boldsymbol{w}_{1}=\boldsymbol{f}_{w 1}\left(\boldsymbol{\Lambda} \hat{\boldsymbol{y}}, \boldsymbol{R}_{D M D}\right)
$$

The control deviation of main controller as follows:

$$
\Delta \hat{\boldsymbol{y}}_{2}=\boldsymbol{y}_{2}-\boldsymbol{f}_{\boldsymbol{y}}\left(\boldsymbol{R}_{D M D}\right)
$$

The link among main regulator, associate regulator, state feedback based on IFO-KAx, intelligent prediction control and BTU compensation as follows:

$$
\left\{\begin{array}{l}
\Delta \hat{\boldsymbol{y}}_{1}=\tilde{\boldsymbol{k}}_{\mathrm{BTU}} \cdot \boldsymbol{y}_{1}-\boldsymbol{w}_{2} \\
\boldsymbol{w}_{2}=-\boldsymbol{w}+\Delta \hat{\boldsymbol{y}}_{2 P I D}+\boldsymbol{w}_{1} \\
\boldsymbol{v}=\Delta \hat{\boldsymbol{y}}_{1 P I D}
\end{array}\right.
$$

An observed system is n-dimensional, controllable, linear, slow time-variant and multi-output. The time-variant characteristics Hammerstein model can be expressed by it and a group high order polynomial which represents nonlinear gain simultaneously according to the feed water control system for super-critical power generation unit.

$$
\begin{aligned}
& \left\{\begin{array}{l}
\dot{\boldsymbol{x}}=\boldsymbol{A}_{\mathrm{W}}(t) \boldsymbol{x}+\boldsymbol{B}_{\mathrm{W}}(t) \boldsymbol{u}_{\mathrm{W}}, \quad \boldsymbol{x}\left(t_{0}\right)=\boldsymbol{x}_{\boldsymbol{0}} \\
\boldsymbol{y}_{\mathrm{W}}=\boldsymbol{C}_{\mathrm{W}}(t) \boldsymbol{x}
\end{array}\right. \\
& \boldsymbol{u}_{\mathrm{W}}=\boldsymbol{f}_{\mathrm{W}_{\boldsymbol{v}}}\left(\boldsymbol{v}_{\mathrm{W}}\right)
\end{aligned}
$$

And the state equation as follows:

$$
\left\{\begin{aligned}
\dot{\boldsymbol{z}} & =\boldsymbol{F}_{\mathrm{W}} \boldsymbol{z}+\boldsymbol{G}_{\mathrm{W}} \Delta \hat{\boldsymbol{y}}_{\mathrm{W}}+\boldsymbol{H}_{\mathrm{w}} \Delta \hat{\boldsymbol{u}}_{\mathrm{W}}, \boldsymbol{z}\left(t_{\boldsymbol{o}}\right)=\boldsymbol{z}_{0} \\
\boldsymbol{w}_{\mathrm{W}} & =\boldsymbol{M}_{\mathrm{W}} \boldsymbol{z}+\boldsymbol{N}_{\mathrm{w}} \Delta \hat{\boldsymbol{y}}_{\mathrm{W}} \\
\Delta \hat{\boldsymbol{u}}_{\mathrm{W}} & =\boldsymbol{v}_{\mathrm{W}}-\boldsymbol{f}_{\mathrm{W} \boldsymbol{v}}\left(\boldsymbol{R}_{\mathrm{W} D M D}\right) \\
\Delta \hat{\boldsymbol{y}}_{\mathrm{W}} & =\boldsymbol{y}_{\mathrm{W}}-\boldsymbol{f}_{\mathrm{W} y}\left(\boldsymbol{R}_{\mathrm{W} D M D}\right)
\end{aligned}\right.
$$
$\mathrm{K} \Delta \mathrm{x}$.

That can express a simultaneous system(9)-(10) IFO-

The regulator of feed water control system as follows:

$$
\begin{aligned}
& \left\{-\Delta \hat{\boldsymbol{y}}_{\mathrm{W}_{1 P I D}}=\boldsymbol{I} \beta_{11} \cdot \Delta \hat{\boldsymbol{y}}_{\mathrm{w}_{1}}+\boldsymbol{I} \beta_{12} \cdot \boldsymbol{z}_{\mathrm{W}_{1}}+\boldsymbol{I} \beta_{13} \cdot \Delta \dot{\hat{\boldsymbol{y}}_{\mathrm{w}}}\right. \\
& \dot{\boldsymbol{z}}_{\mathrm{W}_{1}}=(k+1) \cdot \Delta \hat{\boldsymbol{y}}_{\mathrm{W}_{1}}, \quad \boldsymbol{z}_{\mathrm{W}_{1}}\left(t_{\boldsymbol{\theta}}\right)=\boldsymbol{z}_{\mathrm{W}_{10}}
\end{aligned}
$$

The control deviation of feed water regulator as follows:

$$
\Delta \hat{\boldsymbol{y}}_{\mathrm{W}_{1}}=\boldsymbol{y}_{\mathrm{w}_{1}}-\boldsymbol{f}_{\mathrm{W} \boldsymbol{y}_{1}}\left(\boldsymbol{R}_{\mathrm{W} D M D}\right)
$$

The algorithms which can improve the speed of control system and the dynamic characteristics of system is called generalized intelligent predictive control, the dynamic process 1 and $\mathrm{f} 1(\mathrm{x})$ in figure 1 can be expressed by the following formula:

$$
\boldsymbol{w}_{\mathrm{w} 1}=\boldsymbol{f}_{W w 1}\left(\boldsymbol{w}_{2}\right)
$$

The link among regulator of feed water control system, state feedback based on IFO-K $\Delta \mathrm{x}$, generalized intelligent predictive control and boiler-turbine coordinated control system as follows:

$$
\boldsymbol{v}_{\mathrm{W}}=-\boldsymbol{w}_{\mathrm{W}}+\Delta \hat{\boldsymbol{y}}_{\mathrm{W} 1 P I D}+\boldsymbol{w}_{\mathrm{w} 1}
$$

Inference1: the control system is composed with observability simultaneous system(9)-(15), and the close loop-control is asymptotical stabilization, if it meets the following sufficient condition.

(1) $\operatorname{Re} \lambda\left(\boldsymbol{F}_{\mathrm{w}}\right)<0$

$$
\begin{aligned}
& \text { (2) }\left\{\begin{array}{l}
\lim _{t \rightarrow \infty} \Delta \hat{\boldsymbol{u}}_{\mathrm{W}}=\lim _{t \rightarrow \infty}\left[\boldsymbol{v}_{\mathrm{W}}-\boldsymbol{f}_{\mathrm{W} \boldsymbol{v}}\left(\boldsymbol{R}_{\mathrm{W} D M D}\right)\right]=\xi_{1} \\
\left\|\xi_{1}\right\|_{\infty} \leq \omega_{1} \\
\lim _{t \rightarrow \infty} \boldsymbol{v}_{\mathrm{W}}=\gamma
\end{array}\right. \\
& \text { (3) }\left\{\begin{array}{l}
\lim _{t \rightarrow \infty} \Delta \hat{\boldsymbol{y}}_{\mathrm{W}}=\lim _{t \rightarrow \infty}\left[\boldsymbol{y}_{\mathrm{W}}-\boldsymbol{f}_{\mathrm{W} y}\left(\boldsymbol{R}_{\mathrm{W} D M D}\right)\right]=\xi_{2}=\boldsymbol{I} \boldsymbol{\delta}_{1} \cdot \boldsymbol{C} \Delta \boldsymbol{x} \\
\left\|\xi_{2}\right\|_{\infty} \leq \omega_{2}
\end{array}\right.
\end{aligned}
$$

(4) $\alpha\left[\boldsymbol{A}_{\mathrm{W}}(t)\right]=0$ and $\alpha\left(\boldsymbol{F}_{\mathrm{W}}\right)$ are non-singular(there is no public eigenvalue between matrix $\boldsymbol{A}_{\mathrm{W}}(t)$ and $\boldsymbol{F}_{\mathrm{W}}$ at any moment)

(5) $\left\{\boldsymbol{A}_{\mathrm{W}}(t), \boldsymbol{C}_{\mathrm{W}}(t)\right\}$ is complete observability and $\left\{\boldsymbol{F}_{\mathrm{W}}, \boldsymbol{G}_{\mathrm{w}}\right\}$ is complete controllability at any moment 
6) And the proper order time-variant matrix $\boldsymbol{T}_{\mathrm{w}}(t)$ which meets the following formula is existence.

$$
\begin{gathered}
\boldsymbol{M}_{\mathrm{W}} \boldsymbol{T}_{\mathrm{W}}(t)+\boldsymbol{N}_{\mathrm{W}} \boldsymbol{C}_{\mathrm{W}}(t)=\boldsymbol{K}_{\mathrm{W}}(t) \\
\text { (7) } \lim _{t \rightarrow \infty} \boldsymbol{f}_{\mathrm{W} w 1}\left(\boldsymbol{w}_{2}\right)=\boldsymbol{f}_{\mathrm{W} \boldsymbol{v}}\left(\boldsymbol{R}_{\mathrm{W} D M D}\right)+\lim _{t \rightarrow \infty} \boldsymbol{\Delta} \hat{\boldsymbol{u}} \\
\text { (8) } \lim _{t \rightarrow \infty} \Delta \hat{\boldsymbol{y}}_{\mathrm{W} 1 P I D}=-\boldsymbol{M}_{\mathrm{W}}\left(\boldsymbol{F}_{\mathrm{W}}^{-1} \boldsymbol{H}_{\mathrm{W}} \boldsymbol{I} \xi_{1}+\boldsymbol{F}_{\mathrm{w}}^{-1} \boldsymbol{G}_{\mathrm{W}} \boldsymbol{I} \boldsymbol{\xi}_{2}\right)+\boldsymbol{N}_{\mathrm{W}} \Delta \hat{\boldsymbol{y}}_{\mathrm{W}} \\
\text { Certificate: assume } \\
\boldsymbol{z}_{3}=\boldsymbol{z}+\boldsymbol{F}_{\mathrm{W}}^{-1} \boldsymbol{H}_{\mathrm{W}} \boldsymbol{I} \xi_{1}+\boldsymbol{F}_{\mathrm{W}}^{-1} \boldsymbol{G}_{\mathrm{W}} \boldsymbol{I} \xi_{2} \\
\boldsymbol{w}_{3}=\boldsymbol{w}_{\mathrm{W}}+\boldsymbol{M}_{\mathrm{W}} \boldsymbol{F}_{\mathrm{W}}^{-1} \boldsymbol{H}_{\mathrm{W}} \boldsymbol{I} \xi_{1}+\boldsymbol{M}_{\mathrm{W}} \boldsymbol{F}_{\mathrm{W}}^{-1} \boldsymbol{G}_{\mathrm{W}} \boldsymbol{I} \xi_{2} \\
\quad=\boldsymbol{M}_{\mathrm{W}} \boldsymbol{z}_{3}+\boldsymbol{N}_{\mathrm{W}} \Delta \hat{\boldsymbol{y}}_{\mathrm{W}} \\
\boldsymbol{\varepsilon}=\boldsymbol{z}_{3}-\boldsymbol{T}_{\mathrm{W}}(t) \Delta \boldsymbol{x}, \quad \boldsymbol{e}=\boldsymbol{w}_{3}-\boldsymbol{N}_{\mathrm{W}} \boldsymbol{I} \boldsymbol{\xi}_{2}-\boldsymbol{K}_{\mathrm{W}}(t) \Delta \boldsymbol{x} \\
\text { Notes: } \\
\Delta \hat{\boldsymbol{y}}_{\mathrm{W}}=\boldsymbol{C}_{\mathrm{W}}(t) \Delta \boldsymbol{x}+\boldsymbol{I} \boldsymbol{\delta}_{1} \cdot \boldsymbol{C}_{\mathrm{W}}(t) \Delta \boldsymbol{x}
\end{gathered}
$$

The $\boldsymbol{\delta}_{1}$ is vector composed with the unknown Perturbed element.

We can get formula(20)and(21) from the matrix $\boldsymbol{F}_{\mathrm{W}}$ non-singular of condition(4) in inference1 and formula (9),(11), (16)-(18).

$$
\begin{aligned}
& \dot{\boldsymbol{\varepsilon}}=\boldsymbol{F}_{\mathrm{W}} \boldsymbol{\varepsilon}+\left[\boldsymbol{G}_{\mathrm{W}} \boldsymbol{C}_{\mathrm{W}}(t)+\boldsymbol{F}_{\mathrm{W}} \boldsymbol{T}_{\mathrm{W}}(t)-\boldsymbol{T}_{\mathrm{W}}(t) \boldsymbol{A}_{\mathrm{W}}(t)\right] \Delta \boldsymbol{x} \\
& +\boldsymbol{G}_{\mathrm{W}} \boldsymbol{I} \boldsymbol{\delta}_{1} \cdot \boldsymbol{C}_{\mathrm{W}}(t) \Delta \boldsymbol{x}+\boldsymbol{H}_{\mathrm{W}} \Delta \hat{\boldsymbol{u}}_{\mathrm{W}}-\boldsymbol{T}_{\mathrm{W}} \boldsymbol{B}_{\mathrm{W}}(t) \Delta \boldsymbol{u}_{\mathrm{W}} \\
& -\boldsymbol{H}_{\mathrm{W}} \boldsymbol{I} \xi_{1}-\boldsymbol{G}_{\mathrm{W}} \boldsymbol{I} \xi_{2} \\
& \boldsymbol{e}=\boldsymbol{M}_{\mathrm{W}} \boldsymbol{\varepsilon}+\left[\boldsymbol{N}_{\mathrm{W}}\left(\boldsymbol{I}+\boldsymbol{I} \boldsymbol{\delta}_{1}\right) \boldsymbol{C}_{\mathrm{W}}(t)+\boldsymbol{M}_{\mathrm{W}} \boldsymbol{T}_{\mathrm{W}}(t)\right] \Delta \boldsymbol{x} \\
& -\boldsymbol{N}_{\mathrm{W}} \boldsymbol{I} \boldsymbol{\xi}_{2}-\boldsymbol{K}_{\mathrm{W}}(t) \Delta \boldsymbol{x}
\end{aligned}
$$

If the conditions (4)and(5) in inference1 are established, conclution6.58 in paper[16] and theorem1 in paper[17] show that there is a $n \times n$ solution matrix $\boldsymbol{T}=-[\alpha(\boldsymbol{F})]^{-1} \boldsymbol{U}_{F} \Lambda_{q} \boldsymbol{V}_{A}$ in Sylvester matrix equation $\boldsymbol{G C}(t)=\boldsymbol{T}(t) \boldsymbol{A}(t)-\boldsymbol{F} \boldsymbol{T}(t)$ at any moment. If the conditions (3)and(6) in inference 1 are also established, formula (20) and (21) can be expressed as follows:

$$
\begin{aligned}
& \lim _{t \rightarrow \infty} \dot{\boldsymbol{\varepsilon}}=\boldsymbol{F}_{\mathrm{W}} \boldsymbol{\varepsilon}+\boldsymbol{H}_{\mathrm{W}} \boldsymbol{\Delta} \hat{\boldsymbol{u}}_{\mathrm{W}}-\boldsymbol{T}_{\mathrm{W}} \boldsymbol{B}_{\mathrm{W}}(t) \boldsymbol{f}^{\prime}(\xi) \Delta \boldsymbol{v}_{\mathrm{W}}-\boldsymbol{H}_{\mathrm{W}} \boldsymbol{I} \xi_{1} \\
& \lim _{t \rightarrow \infty} \boldsymbol{e}=\boldsymbol{M}_{\mathrm{W}} \boldsymbol{\varepsilon}
\end{aligned}
$$

Because the conditions (4)and(5) in inference 1 are always established in different time, solution matrix $\boldsymbol{T}(\mathrm{t})$ will change due to a change of $\{\boldsymbol{A}(\mathrm{t}), \boldsymbol{C}(\mathrm{t})\}$, and the feedback matrix $\boldsymbol{K}(\mathrm{t})$ is a function of solution matrix $\boldsymbol{T}(\mathrm{t})$, which is expressed by $\boldsymbol{K}[\boldsymbol{T}(\mathrm{t})]$, then we can get formula(24)and(25) from conditions (1)and(2) in inference1.

$$
\begin{aligned}
& \lim _{t \rightarrow \infty} \boldsymbol{\varepsilon}(t)=\lim _{t \rightarrow \infty}\left[\boldsymbol{z}_{3}(t)-\boldsymbol{T}_{\mathrm{W}}(t) \Delta \boldsymbol{x}(t)\right]=\mathbf{0} \\
& \lim _{t \rightarrow \infty} \boldsymbol{e}(t)=\lim _{t \rightarrow \infty}\left[\boldsymbol{w}_{3}(t)-\boldsymbol{N}_{\mathrm{W}} \boldsymbol{I} \xi_{2}-\boldsymbol{K}_{\mathrm{W}}\left(\boldsymbol{T}_{\mathrm{W}}(t)\right) \Delta \boldsymbol{x}(t)\right]=\mathbf{0}
\end{aligned}
$$

Thus we proved that the observer is stable.

we can get formula(26) from condition(7) in inference1 and formula (14)and(15).

$$
\begin{aligned}
& \lim _{t \rightarrow \infty} \Delta \hat{\boldsymbol{y}}_{\mathrm{W} 1 P I D}=\lim _{t \rightarrow \infty}\left(\boldsymbol{v}_{\mathrm{W}}-\boldsymbol{w}_{\mathrm{W} 1}+\boldsymbol{w}_{\mathrm{W}}\right) \\
& =\lim _{t \rightarrow \infty} \boldsymbol{v}_{\mathrm{W}}-\lim _{t \rightarrow \infty} \boldsymbol{f}_{\mathrm{W} \boldsymbol{v}}\left(\boldsymbol{R}_{\mathrm{W} D M D}\right)-\lim _{t \rightarrow \infty} \Delta \hat{\boldsymbol{u}}+\lim _{t \rightarrow \infty} \boldsymbol{w}
\end{aligned}
$$

The conditions(2)and8) in inference1 are substituted into formula(26).

$$
\begin{aligned}
& -\boldsymbol{M}_{\mathrm{W}}\left(\boldsymbol{F}_{\mathrm{w}}^{-1} \boldsymbol{H}_{\mathrm{W}} \boldsymbol{I} \xi_{1}+\boldsymbol{F}_{\mathrm{w}}^{-1} \boldsymbol{G}_{\mathrm{W}} \boldsymbol{I} \xi_{2}\right)=\lim _{t \rightarrow \infty} \boldsymbol{M}_{\mathrm{W}} \boldsymbol{z} \\
& \lim _{t \rightarrow \infty} \boldsymbol{z}=-\left(\boldsymbol{F}_{\mathrm{w}}^{-1} \boldsymbol{H}_{\mathrm{W}} \boldsymbol{I} \xi_{1}+\boldsymbol{F}_{\mathrm{w}}^{-1} \boldsymbol{G}_{\mathrm{W}} \boldsymbol{I} \xi_{2}\right) \\
& \lim _{t \rightarrow \infty} \boldsymbol{z}_{3}=\lim _{t \rightarrow \infty}\left(\boldsymbol{z}+\boldsymbol{F}_{\mathrm{w}}^{-1} \boldsymbol{H}_{\mathrm{W}} \boldsymbol{I} \xi_{1}+\boldsymbol{F}_{\mathrm{w}}^{-1} \boldsymbol{G}_{\mathrm{W}} \boldsymbol{I} \xi_{2}\right)=\boldsymbol{0} \\
& \lim _{t \rightarrow \infty} \boldsymbol{T}_{\mathrm{W}}(t) \Delta \boldsymbol{x}=\lim _{t \rightarrow \infty}\left(\boldsymbol{z}_{3}-\boldsymbol{\varepsilon}\right)=\boldsymbol{0}
\end{aligned}
$$

Because $\boldsymbol{T}_{\mathrm{w}}(t)$ isn't equal to 0 , in order to make sure that formula (30) is always true, the following formula is established.

$\lim _{t \rightarrow \infty} \Delta \boldsymbol{x}=\mathbf{0}$

The proved of inference 1 is over.

Inference2: if condition(7) in inference1 becomes the following formula.

$$
\left\{\begin{array}{l}
\lim _{t \rightarrow \infty} \boldsymbol{f}_{\mathrm{W} w 1}\left(\boldsymbol{w}_{2}\right)=\boldsymbol{f}_{\mathrm{W} \boldsymbol{v}}\left(\boldsymbol{R}_{\mathrm{W} D M D}\right)+\boldsymbol{\xi}_{4} \\
\left\|\boldsymbol{\xi}_{4}\right\|_{\infty} \leq \omega_{4}
\end{array}\right.
$$

$\xi_{4}$ is proper dimensional real vector, $\omega_{4}$ is proper small real numbers. When the close loop-control system which is composed by observability simultaneous system(9)-(15) is asymptotical stabilization, the operating point of regulator changes, condition (8) becomes the following relationship.

$$
\begin{aligned}
\lim _{t \rightarrow \infty} \Delta \hat{\boldsymbol{y}}_{\mathrm{w} 1 P I D} & =-\boldsymbol{M}_{\mathrm{w}}\left(\boldsymbol{F}_{\mathrm{w}}^{-1} \boldsymbol{H}_{\mathrm{w}} \boldsymbol{I} \xi_{1}+\boldsymbol{F}_{\mathrm{w}}^{-1} \boldsymbol{G}_{\mathrm{w}} \boldsymbol{I} \boldsymbol{\xi}_{2}\right) \\
& +\boldsymbol{N}_{\mathrm{w}} \Delta \hat{\boldsymbol{y}}_{\mathrm{W}}+\xi_{1}-\xi_{4}
\end{aligned}
$$

Certificate: omission

Considering the effect of feed water control system to boiler-turbine coordinated control system, the dynamic process 2 and $\mathrm{f}_{3}(\mathrm{x})$ in figure 1 can be expressed by the following formula:

$$
\boldsymbol{w}_{\mathrm{W} 2}=\boldsymbol{f}_{W w 2}\left(\boldsymbol{\Delta} \hat{\boldsymbol{y}}_{\mathrm{W} 1 P I D}\right)
$$

The simultaneous relationship formula(8) among main regulator, associate regulator, state feedback based on IFO$\mathrm{K} \Delta \mathrm{x}$, intelligent prediction control and BTU compensation in figure 1 do the following correction.

$$
\left\{\begin{array}{l}
\Delta \hat{\boldsymbol{y}}_{1}=\tilde{\boldsymbol{k}}_{\mathrm{BTU}} \cdot \boldsymbol{y}_{1}-\boldsymbol{w}_{2} \\
\boldsymbol{w}_{2}=-\boldsymbol{w}+\Delta \hat{\boldsymbol{y}}_{2 P I D}+\boldsymbol{w}_{1} \\
\boldsymbol{v}=\Delta \hat{\boldsymbol{y}}_{1 P I D}+\boldsymbol{w}_{\mathrm{W} 2}
\end{array}\right.
$$

Inference3: the close loop-control system is composed by observability simultaneous system(1)-(7)and(35).

In order to meet the sufficient condition of asymptotical stabilization, adding the following conditions in meeting the conditions (1)-9) in Lemma1.

$$
\lim _{t \rightarrow \infty} \boldsymbol{w}_{\mathrm{W} 2}=\lim _{t \rightarrow \infty} \boldsymbol{f}_{W w 2}\left(\boldsymbol{\Lambda} \hat{\boldsymbol{y}}_{\mathrm{W} 1 P I D}\right)=\boldsymbol{0}
$$

Certificate: omission.

The control deviation of feed water regulator and output feedback deviation in IFO-K $\Delta \mathrm{x}$ separately is expressed as follows:

$$
\begin{aligned}
& \Delta \hat{\boldsymbol{y}}_{\mathrm{W} 1}=\boldsymbol{y}_{\mathrm{W} 1}-\boldsymbol{f}_{\mathrm{W} \boldsymbol{y} 1}\left(\boldsymbol{R}_{\mathrm{W} D M D}\right) \\
& \Delta \hat{\boldsymbol{y}}_{\mathrm{W}}=\boldsymbol{y}_{\mathrm{W}}-\boldsymbol{f}_{\mathrm{W} \boldsymbol{y}}\left(\boldsymbol{R}_{\mathrm{W} D M D}\right)
\end{aligned}
$$

Both can not affect the conclusion of inference 1-3. 


\section{PRACTICAL APPLICATIONS IN ENGINEERING}

The figure2 shows the trends of separator outlet temperature, feed water flow, feed fuel command, when load change from 550 350 MW that coordinated and feed water are in automatic mode, it can be seen that the control scheme reach satisfactory control effect where.

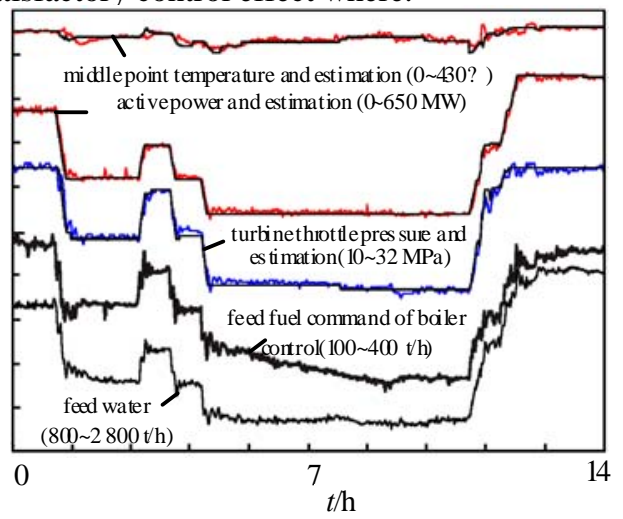

Figure 2. Trends of separator outlet temperature and feed water flow etc. when load change from 550 350 MW

\section{CONCLUSIONS}

The characteristics of super-critical power generation unit coordinated and feed water control system are strong coupling of multiple inputs and multiple outputs, nonlinearity, time-varying parameters. Design object of the study work is that controlled systems are controllable and stable. At first the stability of the coordinated and feed water control system is proved by lemma1 and inference1, secondly the combined operation stability of the coordinated and feed water control system is proved by inference3.

The condition of inference 2 is weaker than inference1, so it is easy to finish in practical engineer, but the load of regulator is heavy.

Mathematical analysis reveals the function of PID regulator in control system, which overcomes the error that is caused by the random deviations $\xi_{1}$ between control system and its mathematical expectation, and the error that is caused by the $\Delta \hat{\boldsymbol{y}}_{\mathrm{W}}\left(\boldsymbol{\xi}_{2}\right)$, and the existence error $\boldsymbol{\xi}_{4}$ between heat of fuel and feed water flow. A theoretical basis and practical method for parameters tuning and error estimates in the practical application of engineer are provided.

\section{REFERENCES}

[1] Zhang Chenyue the mathematical model and dynamic for a boiler[M]. Beijing : Water and electric power press, 1987 : 5461, 94-97.

[2] Zhang Yuduo, Wang Manjia . Automatic control system for thermal[M]. Beijing : Water and electric power press, 1985 : 184190, 190-201.

[3] Yu Daren, Xu Zhiqiang. Supercritical generator unit control and development[J]. Thermal Power Engineering, 2001, 16(2) : 115121.

[4] Fan Yongsheng, Xu Zhigao, Chen Laijiu. Research on modeling and simulation of steam generator of supercritical once-through boiler(No.1)[J]. Proceedings of the CSEE, 1998, 18(4) : 246253.

[5] Fan Yongsheng, Xu Zhigao, Chen Laijiu. Research on modeling and simulation of steam generator of supercritical once-through boiler(No.2)[J]. Proceedings of the CSEE, 1998, 18(5) : 350356.

[6] Wang Wei, Ren Tingjin, Gao Qirui, et al. Mathematical model and simulation of the evaporating surface in a once-through boiler $[\mathrm{J}]$. Journal of Tsinghua University : Science and Technology, 2001, 41(10) : 105-108.

[7] Han Zhongxu, Feng Jian, Qi Xiaohong, et al. Design and application of synthetic coordinated control system to power plant[J]. Control Engineering of China, 2007, 14(4) : 426-429.

[8] Han Zhongxu, Zhou Chuanxin, Zhang Wei, et al. New design method of coordinated control system of supercritical pressure power unit and its application[J]. Proceedings of the CSEE, 2009, 29(8) : 75-81.

[9] Han Zhongxu, Zhou Chuanxin, Li Dan, et al. Soft measurement technology of coal-fired heat and its application on supercritical generating unit control system $[\mathrm{J}]$. Proceedings of the CSEE, 2008, 28(35) : 90-95.

[10] Han Zhongxu. Concept of increment function observer and its application in state feedback control[J]. Proceedings of the CSEE, 2004, 24(4) : 210-216.

[11] Han Zhongxu, Zhang Zhi, Liu Min, et al. Application and design of coordinated control system for No.1\& $2600 \mathrm{MW}$ unit of Beilun power plant[J]. Proceedings of the CSEE, 2006, 26(18) : 126132.

[12] Zhang Chengqian, Zhang Guoqiang. System identification and parameter estimation[M]. Beijing : China Machine PRESS, $1986: 210$.

[13] Zhang Xianda. Matrix analysis and application[M]. Beijing : Tsinghua University press, 2004 : 34-46.

[14] Duan Guangren . Linear system theory[M] . Harbin : harbin institute of technology press, 1996 : 352-356.

[15] Han Zhongxu, Qi Xiaohong, Sun Ying. The method heightening response rate to AGC by analog flexible fuzzy pre-feed coal control and its application[J]. Power System Technology, 2006, 30(s2) : 1-6.

[16] Zheng Dazhong. Linear system theory[M]. second edition , Beijing: Tsinghua University press, 2002 : 342-346.

[17] Han Zhongxu, Qi Xiaohong, Li Dan. Increment function observer with variable gain and its application on time-varying system [J]. Proceedings of the CSEE, 2007, 27(17) : 88-93.

HAN Zhongxu(1957-),male, professor senior engineer, member of Process control professional committee, Chinese Association of Automation, senior member of Chinese Society of Electrical Engineering. He works in Power Plant Technology Research Institute

(Beijing State Power Zhishen Control Technology Ltd.)、Power Grid Digital Simulation Technology Institute and Power Automation Research Institute. His research interests include research and application of computer control system. zhongxuh@epri.sgcc.com.cn

TIAN Xinshou(1985- ), male, PhD student. He works in Power Grid Digital Simulation Technology Institute and Renewable Energy Department of China Electric Power Research Institute one after another. His research interests include research and application of computer control system, and the research on problems of power grid accommodating large scale wind power.

tianxinshou@epri.sgcc.com.cn 